UCRL-JC-124230

PREPRINT

CONF- $960859--5$

\title{
Transient Behavior and Time Lags in Binary Nucleation
}

B. E. Wyslouzil

G. Wilemski

This paper was prepared for submittal to the

\section{RECENED \\ JUN 271996 \\ OSTI}

\section{4th International Conference on \\ Nucleation and Atmospheric Aerosols}

Helsinki, Finland

August 26-30, 1996

April 2, 1996

This is a preprint of a paper intended for publication in a journal or proceedings. Since changes may be made before publication, this preprint is made available with the understanding that it will not be cited or reproduced without the permission of the author.

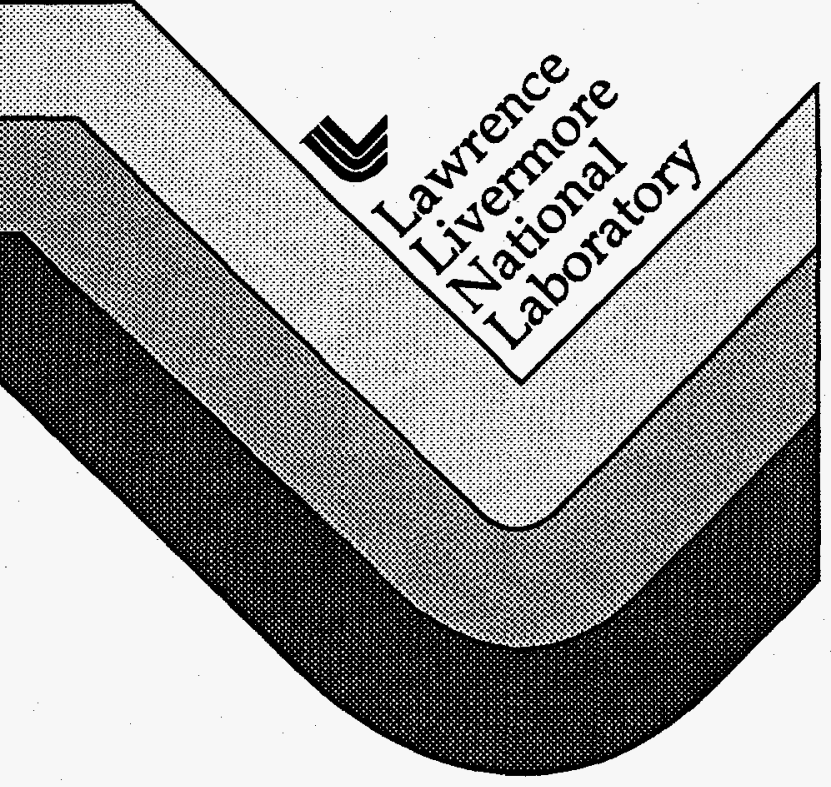




\section{DISCLAIMER}

This document was prepared as an account of work sponsored by an agency of the United States Government. Neither the United States Government nor the University of California nor any of their employees, makes any warranty, express or implied, or assumes any legal liability or responsibility for the accuracy, completeness, or usefulness of any information, apparatus, product, or process disclosed, or represents that its use would not infringe privately owned rights. Reference herein to any specific commercial product, process, or service by trade name, trademark, manufacturer, or otherwise, does not necessarily constitute or imply its endorsement, recommendation, or favoring by the United States Government or the University of California. The views and opinions of authors expressed herein do not necessarily state or reflect those of the United States Government or the University of California, and shall not be used for advertising or product endorsement purposes. 

IN BINARY NUCLEATION

\author{
BARBARA E. WYSLOUZIL \\ Worcester Polytechnic Institute, Worcester, MA 01609-2280 USA \\ and \\ GERALD WILEMSKI \\ Lawrence Livermore National Laboratory, Livermore, CA 94551-9900 USA
}

\begin{abstract}
To investigate transient binary nucleation, both qualitatively and quantitatively, we numerically solved the birth-death equations for vapor-to-liquid phase transitions. We found that in its early transient stages, binary nucleation rarely, if ever, occurs via the saddle point. Instead, most binary systems pass through a temporary stage in which the region of maximum flux extends over a ridge on the free energy surface before reaching the state of saddle point nucleation. Both the number of particles formed and their composition may be affected, and this could be very important for nucleation in glasses and other condensed mixtures for which timescales are very long. In order to plan experiments, accurate estimates of the time lag are important. We therefore directly calculated the time lag for the saddle point flux using our numerical results and compared it with the available analytical predictions. Although the analytical results overestimate the time lag by factors of $2-6$, the numerical results followed the predicted analytical trends quite closely under most conditions.
\end{abstract}

\title{
1. INTRODUCTION
}

Both transient nucleation kinetics and the behavior of the time lags have been investigated in depth in unary systems (see Refs. 1-29 in Wyslouzil and Wilemski, 1996). Much less effort has been expended in examining the transient behavior in binary systems although recently a few numerical studies have appeared (Nishioka and Fujita, 1994; Kožíšek and Demo, 1993, 1995). This paper highlights some of our recent work (Wyslouzil and Wilemski, 1996) in which we numerically solved the coupled kinetics equations describing nucleation in vapor-to-liquid phase transitions. This technique lets us follow the time $(t)$ evolution of any quantity of interest for each cluster composition $(i, j)$, where $i$ and $j$ are the numbers of molecules of species $A$ and $B$, respectively, in the cluster. The objects of interest include the fluxes between adjacent cluster sizes, $J(i, j, t)=J_{A}(i, j, t)+J_{B}(i, j, t)$, the cluster concentrations, $f(i, j, t)$, and the ratios of nonequilibrium to equilibrium cluster concentrations, $\phi(i, j, t)=f(i, j, t) / N(i, j, t)$. In addition we can directly calculate the time lag $t^{*}$ for the saddle point flux. In our paper we examined the transient and time lag behavior of six binary systems representing a wide range of ideal and nonideal solution behavior. Our discussion here focuses on ethanol-hexanol, a system that forms ideal liquid mixtures even though the equilibrium vapor pressures of the pure components differ by a factor of 226 at our simulation temperature of $260 \mathrm{~K}$.

\section{RESULTS AND DISCUSSION}

We solved the kinetics equations describing binary nucleation assuming that the growth and decay of clusters proceeds only by the addition and loss of monomers. The equations, boundary and initial conditions, and computational procedures are presented in two recent papers (Wyslouzil and Wilemski, 1995, 1996) and will not be repeated here. Figure 1 illustrates a typical free energy surface for the ethanol-hexanol system, and Fig. 2 shows how the values of $J(i, j, t)$ evolve to the steady state at these conditions. At the earliest times, Fig. 2(a), the net fluxes are essentially equal to the forward fluxes and the particle fluxes build up most 


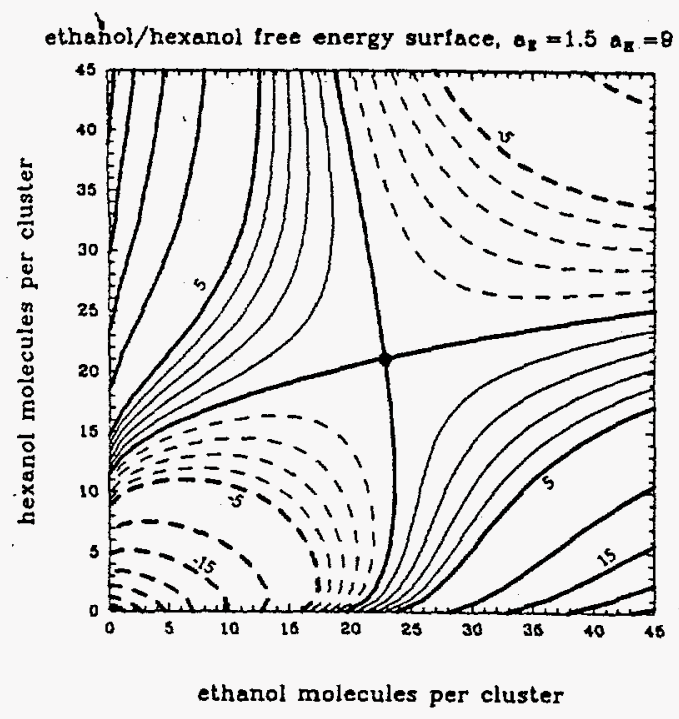

Figure 1. Contour plot of the free energy surface. The analytical saddle point $\bullet$ is located at the junction of the intersecting contours. The contour values are relative to the saddle point free energy, $W^{*} /(k T)=42.4$.
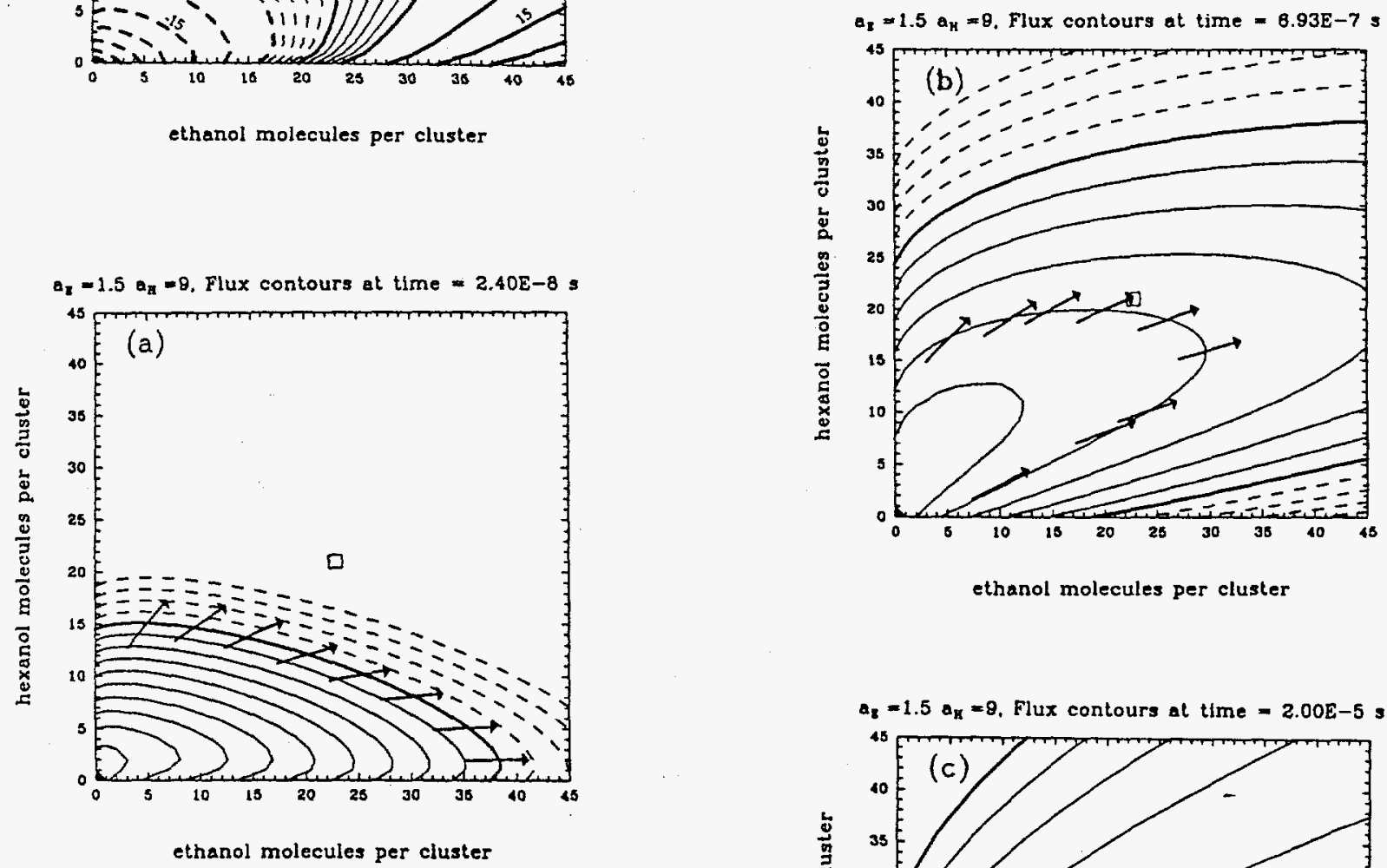

Figure 2. Contours of constant flux, $J(i, j, t)$, at three different times. Heavy contour lines correspond to a flux of $1 \mathrm{~cm}^{-3} \mathrm{~s}^{-1}$. Light solid lines correspond to fluxes greater than $1 \mathrm{~cm}^{-3} \mathrm{~s}^{-1}$ Short dashed lines correspond to fluxes less than $1 \mathrm{~cm}^{-3} \mathrm{~s}^{-1}$. Contour spacing is two orders of magnitude. Arrows indicate the local direction of the flux. The open square marks the saddle point.

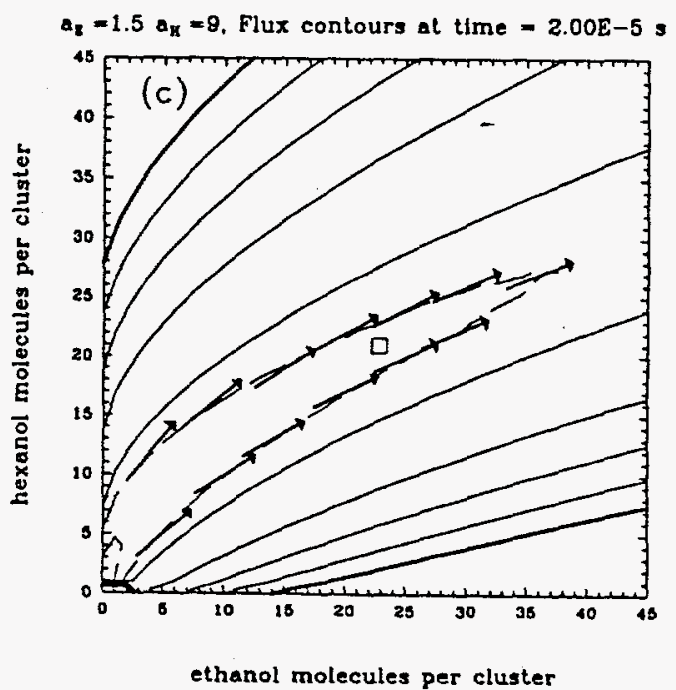

rapidly in the direction of the ethanol axis. Somewhat later, Figs. 2(b) and (c), the fluxes near the pure ethanol axis decrease because the cluster decay steps become important, and the region of highest flux swings toward the saddle. In Fig. 2(b), the region of highest flux extends considerably up the ridge that lies between the saddle point and the pure ethanol axis, and the particle formation process resembles ridge crossing nucleation, cf. Fig. 5(b) of Wyslouzil and Wilemski (1995). In Fig. $2(\mathrm{c}), t \approx 10 \times t^{*}$, and we finally see the relatively narrow steady state nucleation path passing through the saddle point.

As illustrated in Fig. 3, the transient behavior of $\phi(i, j, t)$ presents a much simpler pic- 
$(1, j, t) / N(t, j)$ at time $=2,40 \mathrm{E}-6 \mathrm{~s}$

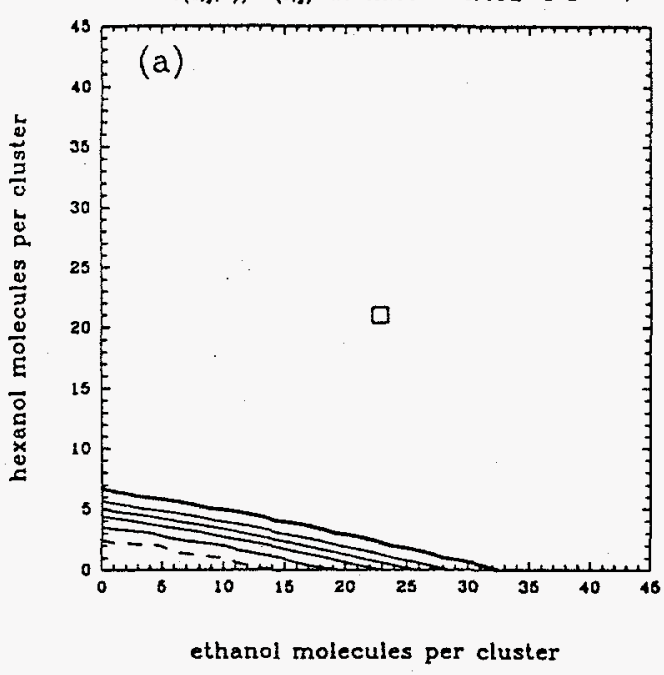

Figure 3. Contours of constant $\phi(i, j, t)$ at three different times. The heavy contour corresponds to $\phi(i, j, t)=0.1$. The light solid lines correspond to higher values and increase in steps of 0.2 . The short dashed line corresponds to $\phi=0.99$. The open square marks the saddle point.
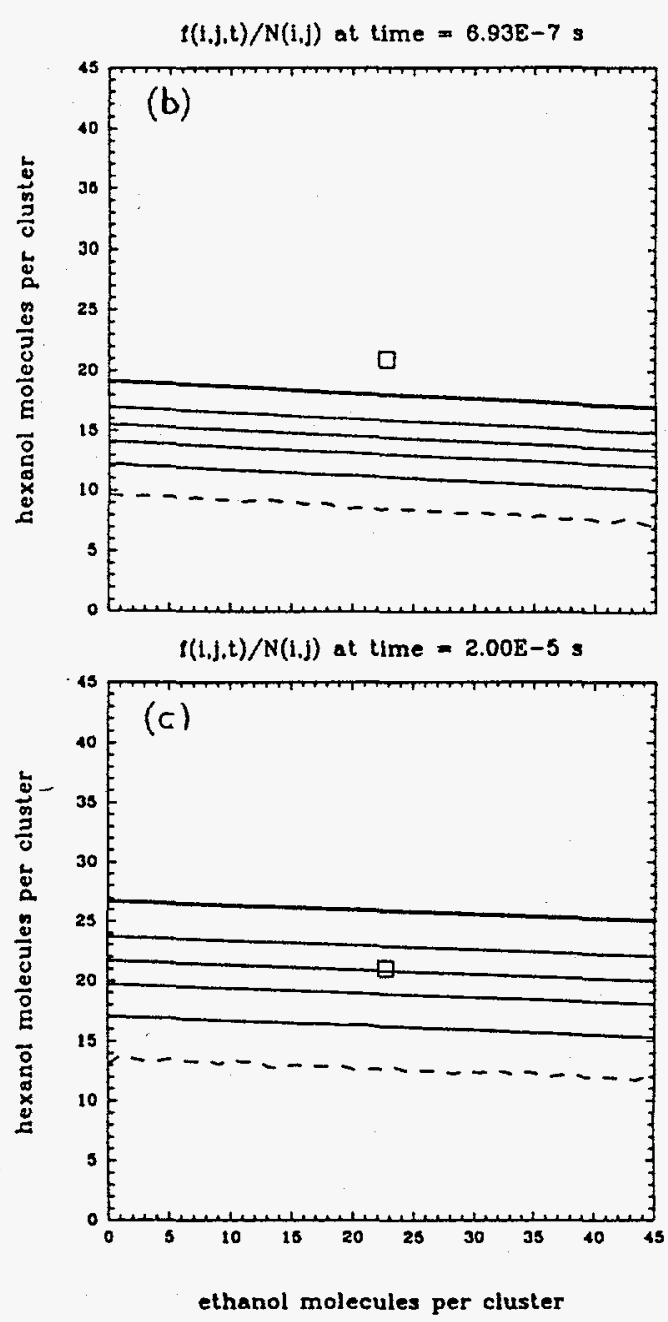

ture. The ideal behavior of the liquid mixture leads to a set of straight parallel contours that progressively moves away from the origin. The difference in timescales for ethanol and hexanol evaporation is responsible for the rapid increase of $\phi$ in the ethanol direction and for the large region of quasi-equilibrium along the ethanol axis. Despite the ideal character of this liquid mixture, this behavior is very similar to that found by Suzuki and Mohnen (1980) for the highly nonideal water-sulfuric acid sytem. As in unary nucleation, the critical cluster concentration equals one half the equilibrium value at steady state. Not illustrated here is the fact that the qualitative behavior of $\phi$ has a "universal" character to it. Changes in the vapor phase activities only weakly affect the time evolution of $\phi$ even in the extreme case when the unary nucleation rate of hexanol (the "slow" component) is higher than the binary rate.

If we define the time lag for the saddle point flux, $t^{*}$, as

$$
t^{*}=\int_{0}^{\infty}\left|1-\frac{J^{*}(t)}{J^{*}}\right| d t,
$$

where $J^{*}(t)=J\left(i^{*}, j^{*}, t\right)$ and $J^{*}$ is the steady state limit of $J^{*}(t)$, we can directly calculate a numerical saddle point time lag $t_{n u m}^{*}$ and compare it to available analytical results for this quantity.

There are only two analytical expressions available for the time lag in binary nucleation. The first, denoted by $t_{W}^{*}$, is the estimate for the time lag at the saddle point developed by Wilemski (1975). The second expression, denoted by $t_{S S}^{*}$, was derived by Shi and Seinfeld (1990). Both expressions have the form $t^{*}=\tau L$ where $\tau$ is a characteristic time scale for binary nucleation determined by the collision frequency of the monomers with a critical cluster and $L$ 
Figure 4. Analytical estimates for $t^{*}$ are compared with the values of $t_{n u m}^{*}$ (solid squares) for ethanol-hexanol at the indicated vapor activities. The light solid lines are $t_{W}^{*}$ using a fit to the values of $L$ given by Wilemski(1975). The heavy solid lines are $t_{W}^{*}$ using the asymptotic result of Shneidman and Weinberg (1992), $2 L=\ln \left(W^{*} / 3 k T\right)+0.5772 \ldots$. The heavy dashed lines are $t_{S S}^{*}$.

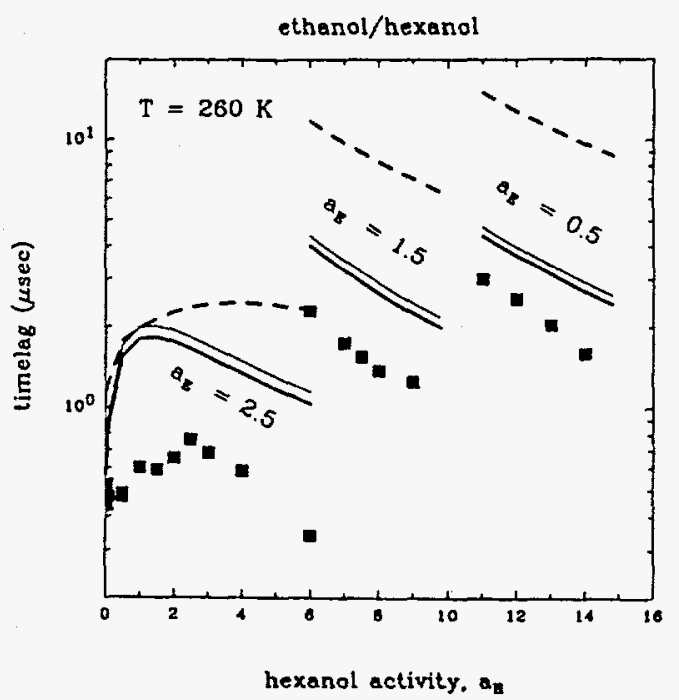

is primarily a function of the dimensionless barrier height $W^{*} / k T$, where $W^{*}$ is the reversible work required to form a critical cluster from the gas phase.

Figure 4 compares our numerical time lag results with the two analytical time lag predictions for the ethanol-hexanol system for a wide range of vapor phase activities. We see that the numerical results follow the analytically predicted trends quite closely although all analytical results are somewhat high.

\section{ACKNOWLEDGMENTS}

This work was supported by the National Science Foundation under Grant No. CHE9502604 (BEW) and the U.S. Department of Energy, Office of Basic Energy Sciences, Division of Geosciences and Engineering (GW). Part of this work was performed under the auspices of the U.S. Department of Energy by the Lawrence Livermore National Laboratory under Contract No. W-7405-ENG-48.

\section{REFERENCES}

Kožíšek, Z. and Demo, P. (1993) Transient kinetics of binary nucleation, J. Cryst. Growth 132, 491.

Kož́šsek, Z. and Demo, P. (1995) Transient nucleation in binary ideal solution, J. Chem. Phys. 102,7595 .

Nishioka, K. and Fujita, K. (1994) Transient binary nucleation of water and sulfuric acid, $J$. Chem. Phys. 100, 532.

Shi, G. and Seinfeld, J. H. (1990) Kinetics of binary nucleation: Multiple pathways and the approach to stationarity, J. Chem. Phys. 93, 9033.

Shneidman, V. A. and Weinberg, M. C. (1992), Induction time in transient nucleation theory, J. Chem. Phys. 97, 3621.

Suzuki, K. and Mohnen, V. A. (1981) Binary homogeneous nucleation theory by cluster-cluster interaction with application to the $\mathrm{H}_{2} \mathrm{SO}_{4}-\mathrm{H}_{2} \mathrm{O}$ system, J. Aerosol Sci. 12, 61 .

Wilemski, G. (1975) Binary nucleation. II. Time lags, J. Chem. Phys. 62, 3772.

Wyslouzil, B.E. and Wilemski, G. (1995) Binary nucleation kinetics. II. Numerical solution of the birth-death equations, J. Chem. Phys. 103, 1137.

Wyslouzil, B.E. and Wilemski, G. (1996) Binary nucleation kinetics. III. Transient behavior and time lags, J. Chem. Phys., submitted 


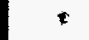



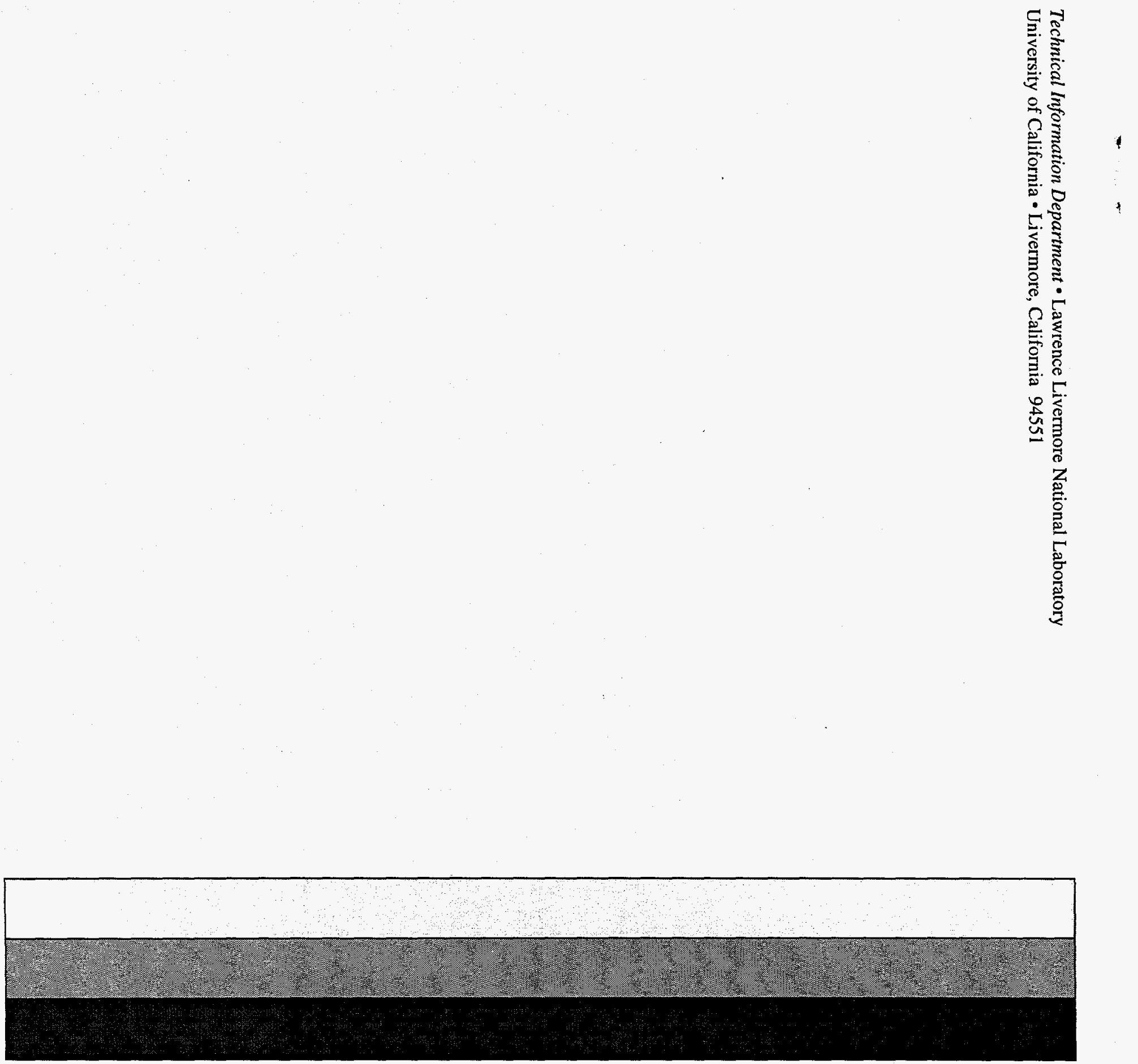